\title{
CONVEX DISTRIBUTION OF THE ZEROS OF STURM-LIOUVILLE FUNCTIONS*
}

BY EINAR HILLE

Introduction. In an investigation concerning the distribution in the complex plane of the zeros of the functions of the parabolic cylinder the author of the present note was confronted with the following problem. Given a linear differential equation of the second order of the type

$$
w^{\prime \prime}+G(z) w=0,
$$

where $G(z)$ is analytic in the region under consideration, how does the nature of the argument of $G(z)$ affect the distribution of the zeros of a given solution $w(z)$ of (1)?

We have some results which throw light on this general question. Roughly speaking, one may say that the argument of $G(z)$ affects the orientation of the zeros in the complex plane, whereas the absolute value of $G(z)$ seems to affect the density of the distribution. As the problem and the results obtained seem to have some intrinsic value aside from their bearing on the special problem, mentioned above, their separate publication may perhaps be justified.

1. Bounded Argument of $G(z)$. Let us consider a convex region $B$ in the $z$-plane in which $G(z)$ is single-valued and analytic and in which, furthermore, the argument of $G(z)$ is restricted as follows:

$$
\vartheta_{1} \geqq \arg G(z) \geqq \vartheta_{2} \quad\left(\pi>\vartheta_{1}-\vartheta_{2} \geqq 0\right) .
$$

Let us construct a set of polygonal lines $(p)$ in $B$ in the following manner. Take any point $P_{0}$ in the interior of $B$. Draw from this point all rays which form an angle $\theta_{P_{0}}$ with the positive real axis such that

$$
-\frac{1}{2} \vartheta_{1}<\theta_{P_{0}}<-\frac{1}{2} \vartheta_{2} \text {. }
$$

Take a second point $P_{1}$ in $B$ on any of these rays and use it as vertex for a second set of rays with the same limitation on the slope-angle $\theta_{P_{1}}$ as on $\theta_{P_{0}}$. Then choose a third point $P_{2}$ and so on. The polygonal line so obtained forms an ele-

\footnotetext{
* Presented to the Society, Dec. 29, 1921.
} 
ment $p$ of our set $(p)$. Repeat this construction for all interior points $P_{0}$ in $B$ and for every possible choice of points $P_{1}, P_{2}, \cdots, P_{n}, \cdots$ on the resulting rays. The set of all these polygonal lines is the set $(p)$. By the construction we define a positive direction on the line $p$, namely from $P_{n}$ to $P_{n+1}$ for all values of $n$; we always assume the line to be traced in this direction. Now take a solution $w(z)$ of $(1)$ and put $W(z)=w(z)(d w / d z)$. Mark the zeros of $W(z)$ in $B$. Then we have the following theorem.

Theorem I. There is at least one polygonal line $p_{0}$ in the set $(p)$, defined above, which joins all the zeros of $W(z)$ in $B$. The line $p_{0}$ is unique when and only when $G(z)=$ const.

The theorem is equivalent to the following one.

Theorem II. If $a$ and $b$ are two zeros of $W(z)$ in $B$, then where $k$ is 0 or 1 .

$$
-\frac{1}{2} \vartheta_{1}+k \pi \leqq \arg (a-b) \leqq-\frac{1}{2} \vartheta_{2}+k \pi,
$$

We have proved theorem II in another place* but the proof will be repeated for the benefit of the reader. Multiply equation (1) by $\bar{w}$, the conjugate of $w$, and integrate between $a$ and $b$. After an integration by parts we obtain

$$
\left[\bar{w} \frac{d w}{d z}\right]_{a}^{b}-\int_{a}^{b}\left|\frac{d w}{d z}\right|^{2} \overline{d z}+\int_{a}^{b}|w|^{2} G(z) d z=0 .
$$

But $W(z)=0$ for $z=a$ and $b$, hence the integrated part disappears. Putting $z=a+r e^{i \theta}$ where $\theta=\arg (b-a)$, we get

$$
e^{2 i \theta} \int_{0}^{r_{1}}|w|^{2} G(z) d r=\int_{0}^{r_{1}}\left|\frac{d w}{d z}\right|^{2} d r, \quad\left(r_{1}=|b-a|\right)
$$

Thus, denoting the integral on the left-hand side in (5) by $\mathfrak{J}(a, b)$ we observe that $e^{2 i \theta} \mathfrak{J}(a, b)$ is a real positive number.

Hence

$$
2 \theta=2 k \pi-\arg \mathfrak{g}(a, b) .
$$

But by (2) we conclude that $\vartheta_{1} \geqq \arg \mathfrak{d}(a, b) \geqq \vartheta_{2}$, so that

$$
k \pi-\frac{1}{2} \vartheta_{1} \leqq \theta \geqq k \pi-\frac{1}{2} \vartheta_{2},
$$

where the signs of equality hold only when $\vartheta_{1}=\vartheta_{2}$, which cleaily corresponds to $G(z)=$ const., when all the zeros lie on the same straight line.

*On the zeros of Sturm-Liouville functions, theorem XI, ARKIV FöR Matematik, Astronomi och Fysik, vol. 16, nr. 17, 1921. 
2. Monotonic Variation of $\arg G(z)$. The line $p_{0}$ is not unique but the sub-set $\left(p_{0}\right)$ of lines in $(p)$ which pass through all the zeros of $W(z)$ in $B$ meet these points in one and the same order. Suppose we enumerate the zeros $a_{1}, a_{2}, \cdots, a_{n}$ in the order in which they occur on any of these lines. Let $\varpi_{0}$ stand for the polygonal line in $\left(p_{0}\right)$ for which $P_{\nu}=a_{\nu+1}$ $(\nu=0,1, \cdots, n-1)$. We are going to show that if the argument of $G(z)$ varies in a monotonic manner along all lines of $(p)$, then $\varpi_{0}$ together with the line $\left(a_{n}, a_{1}\right)$ encloses a convex polygon. This is more precisely stated as follows.

Theorem III. If $\arg G(z)$ is never increasing (never decreasing) when $z$ traces any line of $(p)$, then the angles arg $\left(a_{\nu+1}-a_{\nu}\right)$ form a never decreasing (never increasing) set.

To fix our ideas, let us assume arg $G(z)$ never increasing and put arg $\left(a_{\nu+1}-a_{\nu}\right)=\theta_{\nu}$. Then we have to prove that $\theta_{\nu} \leqq \theta_{\nu+1}$, for all values of $\nu$. By (6) we have

$$
\left\{\begin{array}{l}
2 \theta_{\nu}=-\arg \mathfrak{d}\left(a_{\nu}, a_{\nu+1}\right), \\
2 \theta_{\nu+1}=-\arg \mathfrak{J}\left(a_{\nu+1}, a_{\nu+2}\right) .
\end{array}\right.
$$

But arg $G(z)$ is never increasing along $\varpi_{0}$, so that

$$
\left\{\begin{array}{l}
\arg G\left(a_{\nu+1}\right) \leqq \arg \mathfrak{d}\left(a_{\nu}, a_{\nu+1}\right) \leqq \arg G\left(a_{\nu}\right) \\
\arg G\left(a_{\nu+2}\right) \leqq \arg \mathfrak{d}\left(a_{\nu+1}, a_{\nu+2}\right) \leqq \arg G\left(a_{\nu+1}\right) .
\end{array}\right.
$$

In view of (8) and (9) we conclude that $\theta_{\nu} \leqq \theta_{\nu+1}$ as asserted.

3. A Zero-free Region. Formula (5) suggests a still more general point of view on the problem in hand. At each point $z$ of the plane where $G(z)$ is analytic and $\neq 0$ there is a critical direction $\theta_{z}$ determined by

$$
\theta_{z}=-\frac{1}{2} \arg G(z)
$$

$z$ and $\theta_{z}$ define a lineal element $p_{z}$. These elements envelop a set of curves $\subseteq$ which are integral curves of the differential equation

$$
\Im \mathfrak{m}[\sqrt{G(z)} d z]=0 .
$$

In fact, from (10) it follows that at the point $z$

$$
\frac{1}{2} \arg G(z)+\arg d z \equiv 0(\bmod \pi),
$$

which is equivalent to (11).

Let us take a point $z_{1}$ where $G(z)$ is analytic and $\neq 0$. Mark the singular points $a_{1}, a_{2}, \cdots, a_{n}, \cdots$ of $G(z)$ and join them with $\infty$ by means of straight lines which produced back- 
wards pass through $z_{1}$. These lines $L_{1}, L_{2}, \cdots, L_{n}, \cdots$ we consider as cuts in the plane.

Take a pencil of rays $l\left(\varphi, z_{1}\right)$ with $z=z_{1}$ as vertex. Let us follow a special ray $l\left(\varphi_{0}, z_{1}\right)$ from $z_{1}$. Note the lineal elements along $l$; if $\varphi_{0} \neq \theta_{z_{1}}$, we proceed until we arrive at a point $z_{2}=z_{2}\left(\varphi_{0}\right)$ where $\theta_{z_{2}} \equiv \varphi_{0}(\bmod \pi)$; and the remainder of the ray we leave out. From the rays that pass through the singular points we leave out at least the lines $L_{\nu}$. The rays $\varphi_{0} \equiv \theta_{z_{1}}$ $(\bmod \pi)$ we follow until we meet a second point $z_{2}$ for which $\theta_{z_{2}} \equiv \varphi_{0}$. The points $z_{2}(\varphi)$ form a certain curve which may lie partly at infinity and in general also has isolated discontinuities. The finite points, except possibly the singular points $a_{\nu}$, form part of an analytic curve $\mathfrak{I}\left(z_{1}\right)$ which is the locus of the points of tangency of the pencil of straight lines through $z=z_{1}$ with the family of curves $\mathfrak{S}$. From the differential equation of the pencil and of the family, namely,

$$
\left\{\begin{array}{l}
\Im \mathfrak{m}[\sqrt{G(z)} d z]=0 \\
\Im \mathfrak{m}\left[d z /\left(z-z_{1}\right)\right]=0
\end{array}\right.
$$

we conclude that the equation of $\mathfrak{T}\left(z_{1}\right)$ is

$$
\Im \mathfrak{m}\left[\sqrt{G(z)}\left(z-z_{1}\right)\right]=0 .
$$

If $z_{2}(\varphi)$ is discontinuous at $\varphi=\varphi_{1}$, we assume the existence of $z_{2}{ }^{-}=\lim z_{2}\left(\varphi_{1}-\epsilon\right)$ and of $z_{2}{ }^{+}=\lim z_{2}\left(\varphi_{1}+\epsilon\right)$, neither of which is required to be finite. If we add the part of the ray $l\left(\varphi_{1}\right)$ from $z_{2}^{-}$to $z_{2}^{+}$, we make the boundary curve continuous at $\varphi=\varphi_{1}$. If $z_{2}\left(\varphi_{\nu}\right)=a_{\nu}$, one of the singular points, we have to complete $z_{2}(\varphi)$ by parts of the edges of the cut $L_{\nu}$, namely, from $a_{\nu}$ to $z_{2}^{-}$and from $a_{\nu}$ to $z_{2}{ }^{+}$respectively. What we add from the cuts we consider as inaccessible parts of the boundary, whereas the additions from the interior of the cut plane as well as the part of $\mathfrak{I}\left(z_{1}\right)$ which belongs to $z_{2}(\varphi)$ are regarded as accessible.

The points on $l(\varphi, z)$ from $z_{1}$ to $z_{2}(\varphi)(0 \leqq \varphi<2 \pi)$ form a region $*\left(z_{1}\right)$ which we shall call the star of $z_{1}{ }^{*}$ We count the accessible part of the boundary as belonging to the star;

* In a paper Oscillation theorems in the complex domain, to appear in the Transactions of this Societr, we have given a different definition of the star. The definition given above is simpler and slightly more general. 
the inaccessible points on the cuts and the vertex $z=z_{1}$ we do not include in $*\left(z_{1}\right)$. Then we have

Theorem IV. If $W(z)=w(z)(d w / d z)$, where $w(z)$ is a solution of (1), and if $W\left(z_{1}\right)=0$, then $W(z)$ does not vanish in the star of $z_{1}$.

The proof is obvious. Suppose $z_{3}$ is a point in $*\left(z_{1}\right)$. Then

$$
(k+1) \pi>2 \arg \left(z-z_{1}\right)+\arg G(z)>k \pi,(k=0 \text { or } 1),
$$

for all values of $z$ on the segment $\left(z_{1}, z_{3}\right)$ and where $k$ only depends upon $z_{3}$. This shows that the integral on the lefthand side in formula (5) with $r_{1}=\left|z_{3}-z_{1}\right|$ cannot be positive, which is necessary if $z=z_{3}$ is to be a zero of $W(z)$.

If $\arg G(z)$ satisfies the restriction (2) in a convex region $B$, then $\theta_{z}$ satisfies the inequality (3); that is to say, the line part of the lineal element $p_{z}$ is a line in $(p)$ as far as the neighborhood of $z$ is concerned. If the variation of the argument of $G(z)$ along lines in $(p)$ is monotonic, then the parts of the curves $\subseteq$ which lie in $B$ are all curved in the same way. In fact the curvature of a curve $\Im_{0}$ is found to be

$$
\frac{1}{r}=-\frac{1}{2} \frac{d \phi}{d s},
$$

where $\phi=\arg G(z)$ and $s$ is the length of arc along $\widetilde{S}_{0}{ }^{*}$ The curve $\widetilde{S}_{0}$ lies to the right of the positive tangent if $(d \phi / d s)>0$, but to the left if $(d \phi / d s)<0$.

Assume, to fix our ideas, $(d \phi / d s)<0$. Then the part of $\mathfrak{I}\left(z_{1}\right)$ that lies in $B$ lies to the left of $p_{z_{1}}$ except at $z=z_{1}$, which is the point of tangency. $\mathfrak{I}\left(z_{1}\right)$ is convex with regard to $p_{z_{1}}$ and cuts the boundary of $B$ in two points only. The points on and to the right of $p_{z_{1}}$ in $B$ belong to $*\left(z_{1}\right)$, and likewise those on and to the left of $\mathfrak{I}\left(z_{1}\right)$.

It is worth noticing that the form of the star depends only upon the argument of $G(z)$. Thus if we replace $G(z)$ by $k^{2} G(z)$, where $k$ is real, we get exactly the same configuration. The frequency of the oscillation is of course changed by such a replacement.

HARVARD UNIVERSITY

\footnotetext{
* The curvature is by definition $d \theta_{z} / d s$.
} 